\title{
CONSUMO ENERGÉTICO DOMICILIARIO RESPONSABLE, CASO VIVIENDA CUSCO
}

\author{
Responsible domestic energetic consumption, Cusco case
}

\author{
André Olivares García 1, \\ ${ }^{1}$ Universidad Andina del Cusco, Cusco, Perú.
}

\section{Resumen}

El presente trabajo, se basó en el desarrollo de una actividad de investigación y responsabilidad social con el fin de promover el consumo energético responsable y el desarrollo sostenible. Para la presente actividad se investigó el consumo de la energía eléctrica de un domicilio en un mes, el mismo que fue sustentado por la teoría de la electricidad realizada en clases de Física que se dicta en la Universidad Andina del Cusco, reforzada con fuentes bibliográficas. Por medio de tablas y recurriendo a la teoría, se determinó el consumo de energía, procediéndose luego, a la comparación del mismo con el total a pagar que se presenta en el recibo de luz de la empresa que suministra la energía eléctrica. Finalmente, se aportaron sugerencias para disminuir este consumo energético en los domicilios en general; además de evaluar las oportunidades que nos podría brindar la energía solar.

Palabras clave: Desarrollo sostenible, consumo energético, energía Solar

\begin{abstract}
The present work, was based in developed of an activity of investigation and social responsibility in order to promote the energetic responsible consumption and the sustainable development. The present activity was investigated, the consumption of the electric power of one month in a domicile and sustained by the theory of the electricity realized in classes of Physics that is develop in Andina University of Cusco, reinforced with bibliographical sources. By between of tables and the base of the theory, the energy consumption decided and proceeding to the comparison of the same one with the total to pay that us show the receipt of light of the company that supplies the electric power. Finally, suggestions were contributed for the diminish from energetic consumption in the domiciles in general, beside evaluating the opportunities that the solar power might offer to us.

Keywords: sustainable development, energetic consumption , solar power
\end{abstract}

Citar como: Olivares, A. (2018). Consumo energético domiciliario responsable, caso vivienda Cusco. Rev Yachay, 7(1),302-309.

Recibido: 16-09-2018; Aceptado 07-12-2018

\section{Introducción}

En la actualidad, la energía eléctrica es realmente indispensable, tan es así, que para nosotros es casi imposible vivir sin ella. La necesitamos para desarrollar nuestras actividades diarias, y mantenernos comunicados, con el uso de, por ejemplo, los celulares que funcionan con energía eléctrica para su activación; además, se utiliza electricidad para el uso de una cocina, microondas, refrigerador, ducha eléctrica, televisión, etc. Observando, tenemos que gran parte de los aparatos que poseemos en nuestros domicilios funcionan con energía eléctrica, los mismos que están hechos para facilitarnos el día a día, permitiendo que casi todo pueda realizarse de una manera más rápida.

Se observa que, millones de personas utilizan la energía eléctrica, pero solo un mínimo de estas sabe en realidad cómo funciona, cuánto se gasta por cada artefacto, como disminuir el consumo y de qué fuentes de energía o alternativas se dispone; es por ello que, el objetivo de este trabajo de investigación es promover y dar a conocer el consumo de energía responsable, dando otras alternativas para reducir el consumo y su costo, especificando los montos y cantidades de energía que gastamos y la posibilidad de disminuirlos, así como evaluar el uso de otra energía alternativa a la energía eléctrica. Es por lo tanto que esta investigación pretende demostrar que al reducir ciertas actividades se disminuirán los costos de electricidad mensual. Finalmente, se pretende que las personas puedan conocer cómo se puede seguir un consumo eléctrico responsable.

Rev Yachay volumen (7) Numero (1), enero-diciembre 2018 


\section{Fundamento teórico \\ Electricidad}

Es una forma invisible de energía que produce como resultado la existencia de unas diminutas partículas llamadas electrones libres en los átomos de ciertos materiales o sustancias. Estas partículas, al desplazarse a través de la materia, constituyen lo que denominamos una corriente eléctrica. Es decir, que es un agente físico que llena la estructura atómica de la materia, y todo lo que vemos, sentimos y ocupa un lugar en el espacio, está constituido por diminutas partículas o corpúsculos de electricidad, denominados electrones. En otras palabras, la electricidad no es un invento del hombre sino una fuerza natural; esta fuerza o fenómeno físico se origina por cargas eléctricas estáticas o en movimiento. Cuando una carga se encuentra en reposo produce fuerzas sobre otras situadas en su entorno. Si la carga se desplaza produce también fuerzas magnéticas (Guerrero, 2009, pág. 4).

\section{Potencia eléctrica}

La potencia eléctrica es la relación de transferencia de energía por unidad de tiempo; es decir, la cantidad de energía entregada o absorbida por un elemento en un tiempo determinado.

La potencia eléctrica determina la velocidad a la cual se consume o se suministra energía en los circuitos eléctricos y electrónicos.

La unidad para potencia es el watt (W) que representa un suministro de energía de un joule por segundo (J/S)(ABEJA, 2010,Potencia Electrica)

\section{Aparatos eléctricos}

Son elementos utilizados para realizar tareas que utilizan corriente eléctrica. Están destinados a ser utilizados con una tensión nominal no superior a 1.000 voltios en corriente alterna y 1.500 voltios en corriente continua y los dispositivos necesarios para generar, transmitir y medir tales corrientes (Bordón, 2003).

Tabla de consumo de artefactos.

Tabla 1

Consumo de artefactos eléctricos

\begin{tabular}{|c|c|c|c|}
\hline Artefacto eléctrico & $\begin{array}{l}\text { Potencia } \\
\text { Eléctrica(Watt) }\end{array}$ & Artefacto eléctrico & $\begin{array}{l}\text { Potencia } \\
\text { Eléctrica(Watt) }\end{array}$ \\
\hline $\begin{array}{l}\text { Cocina eléctrica de } 4 \\
\text { hornillas }\end{array}$ & 4500 & Electrobomba & 375 \\
\hline Ducha eléctrica & 4500 & Refrigeradora & 350 \\
\hline Terma eléctrica & 1500 & Computadora & 300 \\
\hline Secadora & 1200 & Licuadora & 300 \\
\hline Aspiradora & 1200 & TV 20" & 120 \\
\hline Microondas & 1100 & Equipo de sonido & 80 \\
\hline Olla arrocera & 1000 & Ventilador & 50 \\
\hline Plancha & 1000 & Laptop & 35 \\
\hline Cafetera & 800 & DVD & 20 \\
\hline Lavadora & 500 & Celular & 10 \\
\hline
\end{tabular}

NOTA: Tabla con la potencia eléctrica que tendrán diferentes artefactos. Fuente Osinerming

\section{Energía solar}

La energía solar es la energía producida por el sol y que es convertida a energía útil por el ser humano, ya sea para calentar algo o producir electricidad (como sus principales aplicaciones).

Cada año el sol arroja 4 mil veces más energía que la que consumimos, por lo que su potencial es prácticamente ilimitado (CEMAER, 2017).

Rev Yachay volumen (7) Numero (1), enero-diciembre 2018 


\section{Procedimiento experimental}

1. Se completó la tabla a continuación, donde se llenaron los aspectos requeridos, como: la cantidad de artefactos, tipo de artefacto, la potencia por día y las horas utilizadas por día de dichos artefactos.

Tabla 2

Potencia parcial y horas al día utilizadas

\begin{tabular}{|c|c|c|c|}
\hline Cantidad & $\begin{array}{l}\text { Dispositivos } \\
\text { /Artefactos/ } \\
\text { Equipos }\end{array}$ & $\begin{array}{l}\text { Potencia } \\
\text { Parcial } \\
(\text { KW })\end{array}$ & Horas/ día \\
\hline 8 & Foco común & 0.02 & 4 \\
\hline 1 & Computadora & 0.3 & 2.3 \\
\hline 1 & Lava ropa & 0.5 & 1 \\
\hline 1 & $\begin{array}{l}\text { Secador de } \\
\text { cabello }\end{array}$ & 1.2 & 0.3 \\
\hline 1 & $\begin{array}{l}\text { Calentador de } \\
\text { agua }\end{array}$ & 1 & 0.5 \\
\hline 1 & Plancha & 1 & 0.2 \\
\hline 1 & Heladera & 0.35 & 4.8 \\
\hline 1 & Licuadora & 0.3 & 0.2 \\
\hline 2 & Televisor & 0.12 & 3 \\
\hline 1 & Equipo de sonido & 0.08 & 4 \\
\hline 2 & $\begin{array}{l}\text { Lámpara de } \\
\text { escritorio LED }\end{array}$ & 0.008 & 1 \\
\hline 1 & microondas & 1.1 & 0.5 \\
\hline 1 & ducha eléctrica & 3.5 & 0.5 \\
\hline
\end{tabular}

NOTA: Potencia parcial en Kilowatt y horas al día utilizadas por diferentes artefactos eléctricos

\section{Resultados}

1. Se calculó el costo a pagar por dicho consumo con el uso de la tabla y del precio unitario que nos brinda el recibo, otorgado por la empresa Electro Sur

Consumo total mensual $(\mathrm{kW}-\mathrm{H}) * \mathrm{~S} / 0.5945=$ Costo mensual total

$$
239.58(k W-H) \quad * s / 0.5945 \quad=s / 142.43031
$$

Como resultado tenemos que: utilizando la tabla 2, se obtuvo un costo mensual en soles de S/. 142.43031

2. Se calculó el consumo promedio mensual, a partir del gráfico de la evolución de consumo de energía (dicho grafico consta de un gráfico de barras, el cual ocupa 12 meses para mostrar dicha evolución) 


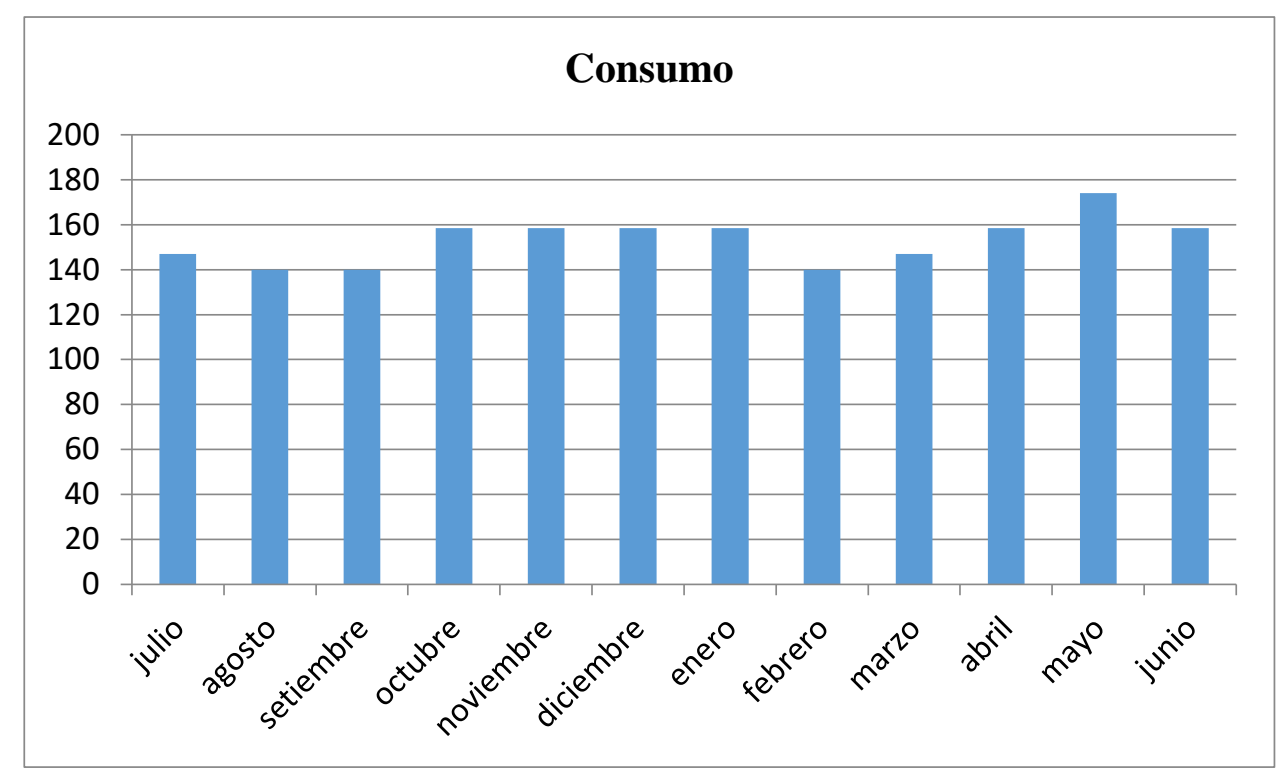

El consumo promedio mensual se puede observar en la tabla $\mathrm{N}^{\circ} 3$.

Tabla 3

Promedio del consumo eléctrico mensual durante un año

\begin{tabular}{ll}
\hline Mes & consumo \\
\hline Julio & 147 \\
\hline Agosto & 140 \\
\hline setiembre & 140 \\
\hline octubre & 158.4 \\
\hline noviembre & 158.4 \\
\hline diciembre & 158.4 \\
\hline Enero & 158.4 \\
\hline febrero & 140 \\
\hline Marzo & 147 \\
\hline Abril & 158.4 \\
\hline Mayo & 174 \\
\hline Junio & 158.4 \\
\hline Sumatoria & \\
\hline Promedio & 1838.4 \\
\hline
\end{tabular}

Nota: Se utilizó el grafico de barras sobre consumo eléctrico mensual para realizar la tabla, con el objetivo de obtener el promedio de este consumo 
Obteniendo:

Un costo mensual promedio de S/. 153.2 utilizando la Tabla $\mathrm{N}^{\circ} 3$

3. Se comparó el consumo energético calculado en el punto 1 y el costo promedio calculado con el recibo de energía proporcionado por el proveedor de energía (Electro sur) en el punto 2.

$$
153.2-142.43031=10.76969
$$

\section{Discusión}

Se dio ideas que se podrían realizar para disminuir el costo de energía en su vivienda (Se realizaron los cambios necesarios y se recalculo su consumo)

En mi hogar actualmente hemos realizado el cambio de focos normales a focos ahorrativos para disminuir el pago mensual por energía eléctrica, además de usar la ducha eléctrica el tiempo justo y necesario que se necesite.

Cambios significativos serían, primero, intercambiar la terma eléctrica por una opción más amigable ecológicamente, esta será reemplazada por una terma solar, siendo esta una de las maneras más eficientes de disminuir el gasto de energía ya que este aparato consume una cantidad significativa de energía. Seguidamente se reducirá el uso de la computadora tomando su uso exclusivamente para trabajos académicos y de investigación, llegando a disminuirlo a 2 horas al día; segundo encender solo los focos necesarios durante la noche (4 focos) y finalmente, el de reducir el uso de los televisores a 1 hora y media, invirtiendo ese tiempo en alguna lectura como libros o periódicos.

Tabla 4

Energía total consumida por mes y su costo en soles

\begin{tabular}{|c|c|c|c|c|c|c|}
\hline Cantidad & $\begin{array}{l}\text { Dispositivos } \\
\text { /Artefactos/ } \\
\text { Equipos }\end{array}$ & $\begin{array}{l}\text { Potencia } \\
\text { Parcial } \\
(\text { KW })\end{array}$ & Horas/ día & $\begin{array}{l}\text { Energía } \\
\text { Total(KW- } \\
\text { H) }\end{array}$ & $\begin{array}{l}\text { Energía } \\
\text { /mes(KW- } \\
\text { H) }\end{array}$ & $\begin{array}{l}\text { costo en soles } \\
(\mathrm{S} / .)\end{array}$ \\
\hline 4 & Foco común & 0.02 & 4 & 0.32 & 9.6 & 5.7072 \\
\hline 1 & Computadora & 0.3 & 2 & 0.6 & 18 & 10.701 \\
\hline 1 & Lava ropa & 0.5 & 1 & 0.5 & 15 & 8.9175 \\
\hline 1 & $\begin{array}{l}\text { Secador } \\
\text { cabello }\end{array}$ & 1.2 & 0.3 & 0.36 & 10.8 & 6.4206 \\
\hline 1 & $\begin{array}{l}\text { Calentador } \mathrm{de} \\
\text { agua }\end{array}$ & 1 & 0.5 & 0.5 & 15 & 8.9175 \\
\hline 1 & Plancha & 1 & 0.2 & 0.2 & 6 & 3.567 \\
\hline 1 & Heladera & 0.35 & 4.8 & 1.68 & 50.4 & 29.9628 \\
\hline 1 & Licuadora & 0.3 & 0.2 & 0.06 & 1.8 & 1.0701 \\
\hline 2 & Televisor & 0.12 & 1.5 & 0.36 & 10.8 & 6.4206 \\
\hline 1 & $\begin{array}{l}\text { Equipo } \\
\text { sonido }\end{array}$ & 0.08 & 4 & 0.32 & 9.6 & 5.7072 \\
\hline 2 & $\begin{array}{l}\text { Lámpara de } \\
\text { escritorio LED }\end{array}$ & 0.008 & 1 & 0.016 & 0.48 & 0.28536 \\
\hline \multirow[t]{2}{*}{1} & microondas & 1.1 & 0.5 & 0.55 & 16.5 & 9.80925 \\
\hline & & & & & 181.68 & 108.00876 \\
\hline
\end{tabular}

NOTA: Esta tabla ya fue modificada y se remarcaron las filas donde hubo cambios.

Rev Yachay volumen (7) Numero (1), enero-diciembre 2018 
Consumo total mensual $(\mathrm{KW}-\mathrm{H}) * \mathrm{~S} / \quad=$ Costo mensual total

$181.68 * 0.5945=108.00876$

Se indagó acerca de la energía solar y qué oportunidades se ven en ella. Este método de obtención de energía genera electricidad a partir del calor del sol el cual es inagotable y renovable, estas energías son obtenidas mediante paneles y espejos.

Viendo cómo funcionan y han sido puestas a prueba durante tantos años (los paneles solares, termas solares, etc.), me llevan a plantear que el uso de la energía solar es el nuevo paso que da la humanidad hacia un mundo de auto sostenibilidad, en casos de edificios auto sostenibles, el uso de los paneles solares y termas solares contribuyen a la auto sostenibilidad al usar esta energía renovable e inagotable que no afecta negativamente al ambiente.

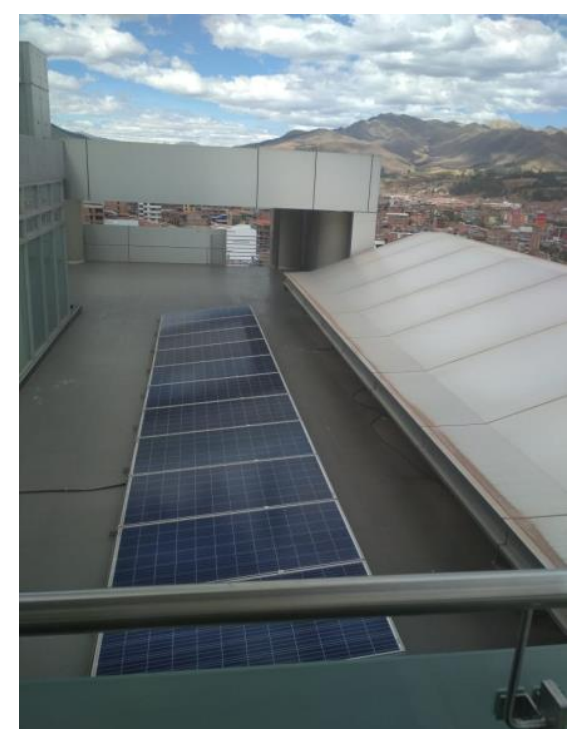

Imagen tomada en el nuevo pabellón de aulas generales de la Universidad Andina del Cusco.

Se hizo una comparación entre la tabla 2 y la tabla 4, para así demostrar el ahorro mensual de dinero que podemos tener al cambiar ciertas cosas en nuestra casa.

Tabla 5

Tabla comparativa para determinar el posible ahorro

\begin{tabular}{|l|l|l|l|l|l|l|}
\hline \multicolumn{2}{|l|}{ Artefactos eléctricos } & Energía /mes & costo en soles & Energía /mes & costo en soles \\
\hline & & $(\mathrm{KW}-\mathrm{H})$ & $(\mathrm{S} /)$. & $(\mathrm{KW}-\mathrm{H})$ & $(\mathrm{S} /)$. \\
\hline Foco común & & 19.2 & 11.4144 & 9.6 & 5.7072 \\
\hline Computadora & & 20.7 & 12.30615 & 18 & 10.701 \\
\hline Lava ropa & & 15 & 8.9175 & 15 & 8.9175 \\
\hline Secador de cabello & & 10.8 & 6.4206 & 10.8 & 6.4206 \\
\hline Calentador de agua & & 15 & 8.9175 & 15 & 8.9175 \\
\hline Plancha & & 6 & 3.567 & 6 & 3.567 \\
\hline
\end{tabular}




\begin{tabular}{|l|l|l|l|l|l|l|}
\hline Heladera & & 50.4 & 29.9628 & & 50.4 & 29.9628 \\
\hline Licuadora & & 1.8 & 1.0701 & & 1.8 & 1.0701 \\
\hline Televisor & & 21.6 & 12.8412 & & 10.8 & 6.4206 \\
\hline Equipo de sonido & & 9.6 & 5.7072 & & 9.6 & 5.7072 \\
\hline Lámpara de escritorio LED & 0.48 & 0.28536 & & 0.48 & 0.28536 \\
& & & & & \\
\hline Microondas & & 16.5 & 9.80925 & 16.5 & 9.80925 \\
\hline Ducha eléctrica & & 52.5 & 31.21125 & & \\
\hline & & SUMATORIA & SUMATORIA & SUMATORIA & SUMATORIA \\
\hline & & 239.58 & 142.43031 & & 181.68 & 108.00876 \\
\hline
\end{tabular}

Nota: Se comparó la energía mensual y el costo en soles entre la tabla $\mathrm{N}^{\circ} 2$ y la $\mathrm{N}^{\circ} 4$, de esta forma se podrá apreciar el ahorro que podemos tener si se modifican ciertos hábitos.

La resta entre los costos de las 2 tablas mencionadas anteriormente nos proporcionará la siguiente información:

$\mathrm{S} / .142 .43031-\mathrm{S} / .108 .00876=\mathrm{S} / .34 .42155$

$\mathrm{Al}$ realizar la comparativa, se puede observar que existe S/. 34.4 de ahorro mensual al cambiar ciertas actividades en la casa.

\section{Discutir sus resultados}

Los resultados con respecto a la tabla y al recibo variarán por diversos factores, como:

El IGV

$>$ Interés moratorio

$>$ Ley de electrificación rural

$>$ Alumbrado público

$>$ Energía ajustada, etc.

Es así como nuestros resultados en base a la tabla varían con respecto al total que percibimos en el recibo.

\section{Conclusiones}

$>$ El uso medido y consciente de nuestros electrodomésticos nos ayuda a gastar menos energía, y eso se refleja en la cantidad total a pagar por mes; quiere decir que, mientras menos tiempo utilicemos nuestros aparatos electrónicos menos pagaremos.

$>$ Se demostró que al cambiar la ducha eléctrica por una solar se redujo el consumo energético y, por lo tanto, hubo reducción en el costo.

$>$ Se demostró que, al reducir el tiempo de ciertas actividades, como, en el uso de la computadora, el televisor y el cambio de focos, esta refleja una disminución en los costos de electricidad mensual.

$>$ En sí, las energías solares, son en su totalidad un elemento sumamente necesario en el nuevo paso de la humanidad hacia la autosostenibilidad, ya que esta es renovable e inagotable.

$>$ La tabla 5, hecha para este informe, varía con los resultados del recibo dado por la compañía de electricidad, debido a que, para esta tabla se aplicaron datos, utilizando la media estadística, es así que no es posible llegar al mismo resultado que en el recibo, a menos que utilicemos la misma tabla que nos proporciona la empresa mediante su página web; aun así, el resultado será aproximado mas no igual al del recibo.

> Como se sabe y se observa, la Universidad Andina del Cusco ya ha puesto un pie adelante sobre el tema de edificaciones autosostenibles con el nuevo pabellón que utiliza energía solar captada por varios paneles solares, demostrando así, el interés que tiene la Universidad Andina del Cusco ante el ambiente.

Rev Yachay volumen (7) Numero (1), enero-diciembre 2018 


\section{Bibliografía}

Abeja, E. K. (16 de Noviembre de 2010). Academia de física de CBTIS $162 . \quad$ Obtenido de https://sites.google.com/site/fisicacbtis162/services/2-5-4-potencia-electrica

Acciona. (s.f.). Obtenido de https://www.acciona.com/es/energias-renovables/energia-solar/

Bordón, L. E. (27 de Enero de 2003). ABC. Obtenido de http://www.abc.com.py/edicion-impresa/suplementos/escolar/aparatoselectricos-622679.html

CEMAER. (2017). gstriatum. Obtenido de http://www.gstriatum.com/energiasolar/

Compara tarifa de energia. (2006). Obtenido de http://www.comparatarifasenergia.es/info-energia/calcular-el-consumo

Electro Sur Este S.A.A. (s.f.). Obtenido de http://www.else.com.pe/else/servicios/SimuleConsumo.aspx

Guerrero, L. E. (2009). La electricidad. En L. E. Guerrero, Electricidad basica I (pág. 4). Machala.

Programa de rehabilitacion de redes electricas. (2013). Obtenido de http://redeselectricasrd.cdeee.gob.do/como-se-mide-el-consumode-energia-electrica/ 